\title{
Matrix spectral norm Wielandt inequalities with statistical applications
}

Jibo $\mathrm{Wu}^{1,2^{*}}$ and $\mathrm{Wende} \mathrm{Yi}^{1,2}$

\section{"Correspondence:}

linfen52@126.com

'School of Mathematics and

Finances, Chongqing University of

Arts and Sciences, Chongqing,

402160, China

2Department of Mathematics and

KLDAIP, Chongqing University of

Arts and Sciences, Chongqing,

402160, China

\section{Abstract}

In this article, we construct a new matrix spectral norm Wielandt inequality. Then we apply it to give the upper bound of a new measure of association. Finally, a new alterative based on the spectral norm for the relative gain of the covariance adjusted estimator of parameters vector is given.

Keywords: Kantorovich inequality; Wielandt inequality; spectral norm

\section{Introduction}

Suppose that $A$ is an $n \times n$ positive definite symmetric matrix, $x$ and $y$ are two nonnull real vectors satisfying $x^{\prime} y=0$ such that

$$
\frac{\left(x^{\prime} A y\right)^{2}}{x^{\prime} A x \cdot y^{\prime} A y} \leq\left(\frac{\lambda_{1}-\lambda_{n}}{\lambda_{1}+\lambda_{n}}\right)^{2}
$$

where $\lambda_{1} \geq \cdots \geq \lambda_{n}>0$ are the ordered eigenvalues of $A$. Inequality (1) is usually called Wielandt inequality in literature; see Drury et al. [1]. Gustafson [2] gave some meaning of this inequality.

Let the random vector $h$ has the covariance matrix $A$, then the maximum of the squared correlation is given as follows:

$$
\max _{x, y: x^{\prime} y=0} \operatorname{corr}^{2}\left(x^{\prime} h, y^{\prime} h\right)=\max _{x, y: x^{\prime} y=0} \frac{\left(x^{\prime} A y\right)^{2}}{x^{\prime} A x \cdot y^{\prime} A y}=\left(\frac{\lambda_{1}-\lambda_{n}}{\lambda_{1}+\lambda_{n}}\right)^{2} .
$$

If we set

$$
y=A^{-1} x-\frac{x^{\prime} A^{-1} x}{x^{\prime} x} x
$$

then the Wielandt inequality (1) becomes the Kantorovich inequality:

$$
\frac{x^{\prime} A x \cdot x^{\prime} A^{-1} x}{\left(x^{\prime} x\right)^{2}} \leq \frac{\left(\lambda_{1}+\lambda_{n}\right)^{2}}{4 \lambda_{1} \lambda_{n}} .
$$

Many authors have been studied the Kantorovich inequality, for more details, see Liu [3, 4], Rao and Rao [5] and Liu and Heyde [6].

O2014 Wu and Yi; licensee Springer. This is an Open Access article distributed under the terms of the Creative Commons Attribution License (http://creativecommons.org/licenses/by/2.0), which permits unrestricted use, distribution, and reproduction in any medium, provided the original work is properly cited. 
Wang and Ip [7] have extended the Wielandt inequality to the matrix version, which can be expressed as follows. Suppose $X$ and $Y$ be $n \times p$ and $n \times q$ matrices satisfying $X^{\prime} Y=0$, then

$$
X^{\prime} A Y\left(Y^{\prime} A Y\right)^{-} Y^{\prime} A X \leq\left(\frac{\lambda_{1}-\lambda_{n}}{\lambda_{1}+\lambda_{n}}\right)^{2} X^{\prime} A X,
$$

where inequality (5) refers to the Loẅner partial ordering.

In inequality (5), $A$ be a positive definite matrix, Lu [8] has extended $A$ to be a nonnegative definite matrix. Drury et al. [1] introduced the matrix, determinant and trace version of the Wielandt inequality. Liu et al. [9] has improved two matrix trace Wielandt inequalities and proposed their statistical applications. Wang and Yang [10] presented the Euclidean norm matrix Wielandt inequality and showed the statistical applications. In this article, we will provide a matrix spectral norm Wielandt inequality and give its application to statistics.

The rest of the article is given as follows. In Section 2, we present a matrix spectral norm versions of the Wielandt inequality. In Section 3, a new measure of association based on the spectral norm is proposed and its upper bound is obtained by using the results in previous section; then we propose an alterative based on the spectral norm of the relative gain of the covariance adjusted estimators of the parameters and its upper bound. Finally, some concluding remarks are given in Section 4.

\section{Matrix spectral norm Wielandt inequality}

We start this section with some notation. Let $A \geq 0$ be an $n \times n$ nonnegative definite matrix of rank $a$ with $a \leq n ; A^{1 / 2}$ is the nonnegative definite square root of $A$; $X$ is an $n \times p$ matrix of rank $k$ with $k \leq p \leq a$; $(\cdot)^{-}$stands for a generalized inverse of a matrix; $(\cdot)^{+}$represents the Moore-Penrose inverse of a matrix; $\operatorname{rank}(\cdot)$ denotes the rank of a matrix; $(\cdot)^{\prime}$ shows for the transpose of a matrix; and $\Re(\cdot)$ stands for the column space of a matrix. Suppose that $P_{A}=A A^{+}$stands for the orthogonal projectors onto the column space of matrix $A$, and use the notation

$$
H=P_{X}=X X^{+}
$$

for the orthogonal projector onto $\Re(X)$.

In order to prove the main results it is necessary to introduce some lemmas.

Lemma 2.1 [4] Let $A \geq 0$ be an $n \times n$ matrix with $\operatorname{rank} a$, and let $X$ be an $n \times p$ matrix of rank $k$ satisfying $\Re(X) \subset \Re(A)$, with $k \leq p \leq a \leq n$. Then

$$
\frac{\operatorname{tr}\left(H A^{-} H\right)^{+}}{\operatorname{tr}(H A H)} \geq\left\{\frac{\sum_{i=1}^{k} 2 \lambda_{i}^{1 / 2} \lambda_{a-i+1}^{1 / 2}}{\sum_{i=1}^{k}\left(\lambda_{i}+\lambda_{a-i+1}\right)}\right\}^{2}
$$

where $\lambda_{1} \geq \cdots \geq \lambda_{a}>0$ are the nonzero eigenvalues of $A$.

Lemma 2.2 [9] If $A \geq 0, \Re(X) \subset \Re(A)$ and $X^{\prime} Y=0$, then

$$
H A Y\left(Y^{\prime} A Y\right)^{+} Y^{\prime} A H \leq H A H-\left(H A^{-} H\right)^{+} .
$$

Lemma 2.3 Let $A \geq 0$ be an $n \times n$ matrix with $\operatorname{rank} a$, and let $X$ be an $n \times p$ matrix of rank $k$ satisfying $\Re(X) \subset \Re(A)$, with $k \leq p \leq a \leq n$. Then $\operatorname{rank}(H A H)=k$. 
Proof As $A$ be a nonnegative definite matrix, we can easily get $\operatorname{rank}(H A H)=\operatorname{rank}(H A)$. By Marsaglia and Styan [11], we have

$$
\operatorname{rank}(H A)=\operatorname{rank}(H)-\operatorname{dim}\left[\Re(H) \cap \Re\left(A^{\perp}\right)\right] .
$$

Since $\Re(X) \subset \Re(A)$, so we have $\Re(H) \subset \Re(A)$, then we obtain $\operatorname{dim}\left[\Re(H) \cap \Re\left(A^{\perp}\right)\right]=0$, that is, $\operatorname{rank}(H A)=\operatorname{rank}(H)$, thus

$$
\operatorname{rank}(H)=\operatorname{rank}(H A H)
$$

On the other hand

$$
\operatorname{rank}(H)=\operatorname{rank}\left(X X^{+}\right)=\operatorname{rank}(X)=k .
$$

So we get $\operatorname{rank}(H A H)=k$.

Lemma 2.4 Let $A \geq 0$ be an $n \times n$ matrix with rank a, and let $X$ be an $n \times p$ matrix of rank $k$ satisfying $\Re(X) \subset \Re(A)$, with $k \leq p \leq a \leq n$. Then

$$
\frac{\left\|\left(H A^{-} H\right)^{+}\right\|_{2}}{\|H A H\|_{2}} \geq \frac{\tau+k-1}{k \tau}\left\{\frac{\sum_{i=1}^{k} 2 \lambda_{i}^{1 / 2} \lambda_{a-i+1}^{1 / 2}}{\sum_{i=1}^{k}\left(\lambda_{i}+\lambda_{a-i+1}\right)}\right\}^{2}
$$

where $\|G\|_{2}=\lambda_{1}(G)$ denotes the spectral norm of the matrix $G, \lambda_{1}(G)$ stands for the largest eigenvalues of matrix $G, \lambda_{1} \geq \cdots \geq \lambda_{a}>0$, are the nonzero eigenvalues of $A, \tau=\frac{\lambda_{1}(H A H)}{\lambda_{k}(H A H)}$ is the condition number of matrix $H A H$.

Proof By the definition of the spectral norm, we obtain

$$
\frac{\left\|\left(H A^{-} H\right)^{+}\right\|_{2}}{\|H A H\|_{2}}=\frac{\lambda_{1}\left(\left(H A^{-} H\right)^{+}\right)}{\lambda_{1}(H A H)} .
$$

By Lemma 2.3, we have $\operatorname{rank}\left(\left(H A^{-} H\right)^{+}\right)=\operatorname{rank}(H A H)=k$, thus we get

$$
\begin{aligned}
\operatorname{tr}\left(\left(H A^{-} H\right)^{+}\right) & =\lambda_{1}\left(\left(H A^{-} H\right)^{+}\right)+\lambda_{2}\left(\left(H A^{-} H\right)^{+}\right)+\cdots+\lambda_{k}\left(\left(H A^{-} H\right)^{+}\right) \\
& \leq \lambda_{1}\left(\left(H A^{-} H\right)^{+}\right)+\lambda_{1}\left(\left(H A^{-} H\right)^{+}\right)+\cdots+\lambda_{1}\left(\left(H A^{-} H\right)^{+}\right) \\
& =k \lambda_{1}\left(\left(H A^{-} H\right)^{+}\right) .
\end{aligned}
$$

So we have

$$
\lambda_{1}\left(\left(H A^{-} H\right)^{+}\right) \geq \frac{1}{k} \operatorname{tr}\left(\left(H A^{-} H\right)^{+}\right) .
$$

On the other hand, define the condition number of the matrix $H A H$ as $\tau=\frac{\lambda_{1}(H A H)}{\lambda_{k}(H A H)}$, then we have

$$
\begin{aligned}
\operatorname{tr}(H A H) & =\lambda_{1}(H A H)+\lambda_{2}(H A H)+\cdots+\lambda_{k}(H A H) \\
& \geq \lambda_{1}(H A H)+\frac{\lambda_{1}(H A H)}{\tau}+\cdots+\frac{\lambda_{1}(H A H)}{\tau}=\frac{\tau+k-1}{\tau} \lambda_{1}(H A H) .
\end{aligned}
$$


Thus,

$$
\lambda_{1}(H A H) \leq \frac{\tau}{\tau+k-1} \operatorname{tr}(H A H)
$$

Then, using Lemma 2.1, we get

$$
\begin{aligned}
\frac{\left\|\left(H A^{-} H\right)^{+}\right\|_{2}}{\|H A H\|_{2}} & =\frac{\lambda_{1}\left(\left(H A^{-} H\right)^{+}\right)}{\lambda_{1}(H A H)} \\
& \geq \frac{\tau+k-1}{k \tau} \frac{\operatorname{tr}\left(\left(H A^{-} H\right)^{+}\right)}{\operatorname{tr}(H A H)} \\
& \geq \frac{\tau+k-1}{k \tau}\left\{\frac{\sum_{i=1}^{k} 2 \lambda_{i}^{1 / 2} \lambda_{a-i+1}^{1 / 2}}{\sum_{i=1}^{k}\left(\lambda_{i}+\lambda_{a-i+1}\right)}\right\}^{2} .
\end{aligned}
$$

Now we present the first theorem of this article.

Theorem 2.1 Suppose $A \geq 0$ to be an $n \times n$ matrix of rank $a$, and suppose $X$ to be an $n \times p$ matrix of rank $k$, and suppose $Y$ to be an $n \times q$ matrix such that $\Re(X) \subset \Re(A)$ and $X^{\prime} P_{A} Y=X^{\prime} Y=0$ with $k \leq p \leq q \leq a \leq n$. Then

$$
\begin{aligned}
& \frac{\left\|H A Y\left(Y^{\prime} A^{-} Y\right)^{+} Y^{\prime} A H\right\|_{2}}{\|H A H\|_{2}} \\
& \leq \frac{\tau+k-1}{k \tau} \frac{\left[\sum_{i=1}^{k}\left(\lambda_{i}^{1 / 2}-\lambda_{a-i+1}^{1 / 2}\right)^{2}\right]\left[\sum_{i=1}^{k}\left(\lambda_{i}^{1 / 2}+\lambda_{a-i+1}^{1 / 2}\right)^{2}\right]}{\left[\sum_{i=1}^{k}\left(\lambda_{i}+\lambda_{a-i+1}\right)\right]^{2}}, \\
& \frac{\left\|H A H-H A Y\left(Y^{\prime} A^{-} Y\right)^{+} Y^{\prime} A H\right\|_{2}}{\|H A H\|_{2}} \geq \frac{\tau+k-1}{k \tau}\left\{\frac{\sum_{i=1}^{k} 2 \lambda_{i}^{1 / 2} \lambda_{a-i+1}^{1 / 2}}{\sum_{i=1}^{k}\left(\lambda_{i}+\lambda_{a-i+1}\right)}\right\}^{2},
\end{aligned}
$$

where $\|G\|_{2}=\lambda_{1}(G)$ denotes the spectral norm of the matrix $G, \lambda_{1}(G)$ stands for the largest eigenvalues of matrix $G, \lambda_{1} \geq \cdots \geq \lambda_{a}>0$ are the nonzero eigenvalues of $A, \tau=\frac{\lambda_{1}(H A H)}{\lambda_{k}(H A H)}$ is the condition number of the matrix $H A H$.

Proof (1) For (19), using Lemma 2.2 and Lemma 2.4, we obtain

$$
\begin{aligned}
\| & H A Y\left(Y^{\prime} A^{-} Y\right)^{+} Y^{\prime} A H \|_{2} \\
& =\lambda_{1}\left(H A Y\left(Y^{\prime} A^{-} Y\right)^{+} Y^{\prime} A H\right) \\
& \leq \lambda_{1}\left(H A H-\left(H A^{+} H\right)^{+}\right) \\
& =\lambda_{1}(H A H)-\lambda_{1}\left(\left(H A^{+} H\right)^{+}\right) \\
& =\|H A H\|_{2}-\left\|\left(H A^{+} H\right)^{+}\right\|_{2} \\
& \leq\|H A H\|_{2}-\frac{\tau+k-1}{k \tau}\left\{\frac{\sum_{i=1}^{k} 2 \lambda_{i}^{1 / 2} \lambda_{a-i+1}^{1 / 2}}{\sum_{i=1}^{k}\left(\lambda_{i}+\lambda_{a-i+1}\right)}\right\}^{2}\|H A H\|_{2} \\
& =\frac{k \tau\left[\sum_{i=1}^{k}\left(\lambda_{i}+\lambda_{a-i+1}\right)\right]^{2}-(\tau+k-1)\left[\sum_{i=1}^{k} 2 \lambda_{i}^{1 / 2} \lambda_{a-i+1}^{1 / 2}\right]^{2}}{k \tau\left[\sum_{i=1}^{k}\left(\lambda_{i}+\lambda_{a-i+1}\right)\right]^{2}}\|H A H\|_{2} .
\end{aligned}
$$


Since $k \geq 1$ and $\tau \geq 1$, then $k \tau-(\tau+k-1)=(k-1)(\tau-1) \geq 0$. Thus we obtain

$$
\begin{aligned}
& \left\|H A Y\left(Y^{\prime} A^{-} Y\right)^{+} Y^{\prime} A H\right\|_{2} \\
& \quad \leq \frac{\tau+k-1}{k \tau} \frac{\left[\sum_{i=1}^{k}\left(\lambda_{i}+\lambda_{a-i+1}\right)\right]^{2}-\left[\sum_{i=1}^{k} 2 \lambda_{i}^{1 / 2} \lambda_{a-i+1}^{1 / 2}\right]^{2}}{\left[\sum_{i=1}^{k}\left(\lambda_{i}+\lambda_{a-i+1}\right)\right]^{2}}\|H A H\|_{2} \\
& \quad=\frac{\tau+k-1}{k \tau} \frac{\left[\sum_{i=1}^{k}\left(\lambda_{i}^{1 / 2}-\lambda_{a-i+1}^{1 / 2}\right)^{2}\right]\left[\sum_{i=1}^{k}\left(\lambda_{i}^{1 / 2}+\lambda_{a-i+1}^{1 / 2}\right)^{2}\right]}{\left[\sum_{i=1}^{k}\left(\lambda_{i}+\lambda_{a-i+1}\right)\right]^{2}}\|H A H\|_{2} .
\end{aligned}
$$

Inequality (19) is proved.

(2) For (20), from Lemma 2.2 and Lemma 2.4, we can obtain

$$
\begin{aligned}
& \left\|H A H-H A Y\left(Y^{\prime} A^{-} Y\right)^{+} Y^{\prime} A H\right\|_{2} \\
& =\lambda_{1}\left(H A H-H A Y\left(Y^{\prime} A^{-} Y\right)^{+} Y^{\prime} A H\right) \\
& \geq \lambda_{1}\left(\left(H A^{+} H\right)^{+}\right) \\
& =\left\|\left(H A^{+} H\right)^{+}\right\|_{2} \\
& \geq \frac{\tau+k-1}{k \tau}\left\{\frac{\sum_{i=1}^{k} 2 \lambda_{i}^{1 / 2} \lambda_{a-i+1}^{1 / 2}}{\sum_{i=1}^{k}\left(\lambda_{i}+\lambda_{a-i+1}\right)}\right\}^{2}\|H A H\|_{2} .
\end{aligned}
$$

The proof of inequality (20) is completed.

Partition matrix $A \geq 0$ as follows:

$$
A=\left(\begin{array}{ll}
A_{11} & A_{12} \\
A_{21} & A_{22}
\end{array}\right), \quad A_{11.2}=A_{11}-A_{12} A_{22}^{-} A_{21},
$$

where $A \geq 0$ of $\operatorname{rank} a, A_{11} \geq 0$ of $\operatorname{rank} k, A_{11}$ is $p \times p$ and $A_{22}$ is $q \times q, p+q=n$.

Now we give another theorem.

Theorem 2.2 Suppose $A$ be an $n \times n$ nonnegative definite matrix of rank a partitioned as in (24) and suppose that

$$
\operatorname{rank}(A)=\operatorname{rank}\left(A_{11}\right)+\operatorname{rank}\left(A_{22}\right)
$$

then

$$
\begin{aligned}
& \frac{\left\|A_{12} A_{22}^{-} A_{21}\right\|_{2}}{\left\|A_{11}\right\|_{2}} \leq \frac{\tau+k-1}{k \tau} \frac{\left[\sum_{i=1}^{k}\left(\lambda_{i}^{1 / 2}-\lambda_{a-i+1}^{1 / 2}\right)^{2}\right]\left[\sum_{i=1}^{k}\left(\lambda_{i}^{1 / 2}+\lambda_{a-i+1}^{1 / 2}\right)^{2}\right]}{\left[\sum_{i=1}^{k}\left(\lambda_{i}+\lambda_{a-i+1}\right)\right]^{2}}, \\
& \frac{\left\|A_{11.2}\right\|_{2}}{\left\|A_{11}\right\|_{2}} \geq \frac{\tau+k-1}{k \tau}\left\{\frac{\sum_{i=1}^{k} 2 \lambda_{i}^{1 / 2} \lambda_{a-i+1}^{1 / 2}}{\sum_{i=1}^{k}\left(\lambda_{i}+\lambda_{a-i+1}\right)}\right\}^{2},
\end{aligned}
$$

where $\lambda_{1} \geq \cdots \geq \lambda_{a}>0$ are the nonzero eigenvalues of $A, \tau=\frac{\lambda_{1}\left(A_{11}\right)}{\lambda_{k}\left(A_{11}\right)}$ is the condition number of matrix $A_{11}$.

Proof (1) Since $A>0$, let the $n \times p$ matrix $X$ be $\left(\begin{array}{c}I_{p} \\ 0\end{array}\right)$ and $n \times q$ matrix $Y$ be $\left(\begin{array}{c}0 \\ I_{q}\end{array}\right)$, then we obtain $X^{\prime} A Y=A_{12}, Y^{\prime} A Y=A_{22}, Y^{\prime} A X=A_{21}, X^{\prime} A X=A_{11}, X^{\prime} X=I_{p}, H=X X^{\prime}, \tau=\frac{\lambda_{1}\left(A_{11}\right)}{\lambda_{k}\left(A_{11}\right)}$, 
$\Re(X) \subset \Re(A)$ and $X^{\prime} P_{A} Y=0$. Substituting it into Theorem 2.1, we can get the two inequalities involving $A_{22}^{-1}$.

(2) As $A \geq 0, A$ can be partitioned as in (24) with $A_{11} \geq 0$ and $A_{22} \geq 0$, then $\Re\left(A_{12}\right) \subset$ $\Re\left(A_{11}\right)$ and $\Re\left(A_{21}\right) \subset \Re\left(A_{22}\right)$. On the other hand, using $\operatorname{rank}(A)=\operatorname{rank}\left(A_{11}\right)+\operatorname{rank}\left(A_{22}\right)$, we get $\Re(X) \subset \Re(A)$, which is needed in Theorem 2.1.

\section{Applications to statistics}

In this section, we give several inequalities involving covariance matrices, an alternative based on the spectral norm of the relative gain of the covariance adjusted estimator and its upper bound by using the inequalities in Section 2 .

\subsection{New measure of association}

Suppose that $\mu$ and $v$ are $p \times 1$ and $q \times 1$ random vectors and that we have the covariance matrix

$$
\operatorname{Cov}\left(\begin{array}{l}
\mu \\
v
\end{array}\right)=\Sigma_{n \times n}=\left(\begin{array}{cc}
\Sigma_{11} & \Sigma_{12} \\
\Sigma_{21} & \Sigma_{22}
\end{array}\right),
$$

where $n=p+q$.

Wang and Ip [7] have discussed the following measure of association for $\Sigma>0$ :

$$
\rho_{1}=\left|\Sigma_{12} \Sigma_{22}^{-1} \Sigma_{21} \Sigma_{11}^{-1}\right|=\frac{\left|\Sigma_{12} \Sigma_{22}^{-1} \Sigma_{21}\right|}{\left|\Sigma_{11}\right|}
$$

where $|\cdot|$ refers to the determinant of the concerned matrix and $p \leq q \leq n$. We can see that $\rho_{1}$ cannot be used when $\left|\Sigma_{11}\right|=0$. As pointed out by Groß [12], the authors may encounter a singular covariance matrix. To solve this problem, Liu et al. [9] introduced a new measure association:

$$
\rho_{2}=\frac{\operatorname{tr}\left(\Sigma_{12} \Sigma_{22}^{-1} \Sigma_{21}\right)}{\operatorname{tr}\left(\Sigma_{11}\right)}
$$

They also gave an upper bound of $\rho_{2}$ and they pointed out that $\rho_{2}$ is useful in canonical correlations and regression analysis areas as discussed by Lu [8], Wang and Ip [7], and Anderson [13].

Wang and Yang [10] presented an alternative measure association, which is defined as follows:

$$
\rho_{3}=\frac{\left\|\Sigma_{12} \Sigma_{22}^{-1} \Sigma_{21}\right\|_{E}}{\left\|\Sigma_{11}\right\|_{E}}
$$

where $\|\cdot\|_{E}$ stands for the Euclidean norm of concerned matrix and they also gave an upper bound of $\rho_{3}$.

As is well known, there is no measure association involving the spectral norm, so we present a new measure of association based on the spectral norm:

$$
\rho_{4}=\frac{\left\|\Sigma_{12} \Sigma_{22}^{-1} \Sigma_{21}\right\|_{2}}{\left\|\Sigma_{11}\right\|_{2}}
$$


Theorem 3.1 The upper bound of $\rho_{4}$ is given as follows:

$$
\rho_{4} \leq \frac{\tau+p-1}{p \tau} \frac{\left[\sum_{i=1}^{p}\left(\lambda_{i}^{1 / 2}-\lambda_{n-i+1}^{1 / 2}\right)^{2}\right]\left[\sum_{i=1}^{p}\left(\lambda_{i}^{1 / 2}+\lambda_{n-i+1}^{1 / 2}\right)^{2}\right]}{\left[\sum_{i=1}^{p}\left(\lambda_{i}+\lambda_{n-i+1}\right)\right]^{2}} \leq 1
$$

where $\lambda_{1} \geq \cdots \geq \lambda_{n}>0$ are the ordered eigenvalues of $\Sigma, \tau=\frac{\lambda_{1}(\Sigma)}{\lambda_{p}(\Sigma)}$ is the condition number of matrix $\Sigma$.

Proof It is easy to prove inequality (32) by using Theorem 2.2 and (31).

\subsection{Wishart matrices}

Let $S$ be an estimator of $\Sigma$, partitioned $S$ as follows:

$$
S=\left(\begin{array}{ll}
S_{11} & S_{12} \\
S_{21} & S_{22}
\end{array}\right), \quad S_{11.2}=S_{11}-S_{12} S_{22}^{-1} S_{21}
$$

where $S_{11}$ is a $p \times p$ matrix.

Wang and Ip [7] presented these interesting relations among these submatrices occurring in much of the statistical literature, such as in linear models

$$
S_{12} S_{22}^{-1} S_{21} \leq \frac{\left(\lambda_{1}-\lambda_{n}\right)^{2}}{\left(\lambda_{1}+\lambda_{n}\right)^{2}} S_{11}
$$

where $\lambda_{1}$ and $\lambda_{n}$ refer to the largest and smallest eigenvalues of $S$, respectively. They also considered the concept of the relative gain of the covariance adjusted estimator of a parameter vector discussed by Rao [14] and Wang and Yang [15]. $\frac{\left|\Sigma_{12} \Sigma_{22}^{-1} \Sigma_{21}\right|}{\left|\Sigma_{11}\right|}$ can be regarded as the relative gain and it can be estimated by $\frac{\left|S_{12} S_{22}^{-1} S_{21}\right|}{\left|S_{11}\right|}$. Liu et al. [9] use $\frac{\operatorname{tr}\left(S_{12} S_{22}^{-1} S_{21}\right)}{\operatorname{tr}\left(S_{11}\right)}$ to estimate $\frac{\operatorname{tr}\left(\Sigma_{12} \Sigma_{22}^{-1} \Sigma_{21}\right)}{\operatorname{tr}\left(\Sigma_{11}\right)}$ and they also showed that

$$
\frac{\operatorname{tr}\left(S_{12} S_{22}^{-1} S_{21}\right)}{\operatorname{tr}\left(S_{11}\right)} \leq \frac{\left[\sum_{i=1}^{p}\left(\lambda_{i}^{1 / 2}-\lambda_{n-i+1}^{1 / 2}\right)^{2}\right]\left[\sum_{i=1}^{p}\left(\lambda_{i}^{1 / 2}+\lambda_{n-i+1}^{1 / 2}\right)^{2}\right]}{\left[\sum_{i=1}^{p}\left(\lambda_{i}+\lambda_{n-i+1}\right)\right]^{2}} \leq 1,
$$

where $\lambda_{1} \geq \cdots \geq \lambda_{n}>0$ are the ordered eigenvalues of $S$.

Wang and Yang [7] also studied this problem and used $\frac{\left\|S_{12} S_{22}^{-1} S_{21}\right\|_{E}}{\left\|S_{11}\right\|_{E}}$ to estimate $\frac{\left\|\Sigma_{12} \Sigma_{22}^{-1} \Sigma_{21}\right\|_{E}}{\left\|\Sigma_{11}\right\|_{E}}$; they also gave an upper bound of $\frac{\left\|S_{12} S_{2}^{-1} S_{21}\right\|_{E}}{\left\|S_{11}\right\|_{E}}$, which is given as follows:

$$
\frac{\left\|S_{12} S_{22}^{-1} S_{21}\right\|_{E}}{\left\|S_{11}\right\|_{E}} \leq \frac{l(h, p)}{2 p}\left(\sqrt{\frac{\lambda_{1} \lambda_{n-p+1}}{\lambda_{p} \lambda_{n}}}+\sqrt{\frac{\lambda_{p} \lambda_{n}}{\lambda_{1} \lambda_{n-p+1}}}\right) \sum_{i=1}^{p} \frac{\lambda_{i}}{\lambda_{n}-p+i},
$$

where $\operatorname{rank}\left(S_{12} S_{22}^{-1} S_{21}\right)=h$ and $l(h, p)=\frac{\max _{h} \sum_{i=1}^{h} \lambda_{i}^{2}}{\max _{p} \sum_{i=1}^{p} \lambda_{i}^{2}}$.

In this article we will present the spectral norm operator instead of the determinant, trace, and Euclidean norm. The new relative gain of the covariance adjusted estimator is denoted by

$$
\omega=\frac{\left\|\Sigma_{12} \Sigma_{22}^{-1} \Sigma_{21}\right\|_{2}}{\left\|\Sigma_{11}\right\|_{2}}
$$


and $\omega$ is estimated by

$$
\hat{\omega}=\frac{\left\|S_{12} S_{22}^{-1} S_{21}\right\|_{2}}{\left\|S_{11}\right\|_{2}}
$$

Now we give the upper bound of $\omega$.

Theorem 3.2 The relative gain $\hat{\omega}$ is bounded as follows:

$$
\hat{\omega} \leq \frac{\tau+p-1}{p \tau} \frac{\left[\sum_{i=1}^{p}\left(\lambda_{i}^{1 / 2}-\lambda_{n-i+1}^{1 / 2}\right)^{2}\right]\left[\sum_{i=1}^{p}\left(\lambda_{i}^{1 / 2}+\lambda_{n-i+1}^{1 / 2}\right)^{2}\right]}{\left[\sum_{i=1}^{p}\left(\lambda_{i}+\lambda_{n-i+1}\right)\right]^{2}} \leq 1,
$$

where $\lambda_{1} \geq \cdots \geq \lambda_{n}>0$ are the ordered eigenvalues of $S, \tau=\frac{\lambda_{1}(S)}{\lambda_{p}(S)}$ is the condition number of matrix $S$.

Proof Using Theorem 2.2, we can easily get the proof of Theorem 3.2.

Remark 3.1 The result in Theorem 3.2 can be extended to the nonnegative definite matrix $S \geq 0$, but $S_{11} \neq 0$.

\section{Concluding remarks}

In this article, we have presented two matrix spectral norm Wielandt inequalities and some applications of the spectral norm Wielandt inequalities, and we also can see that these applications are meaningful, useful, and practical in statistics.

\section{Competing interests}

The authors declare that they have no competing interests.

Authors' contributions

All authors read and approved the final manuscript.

\section{Acknowledgements}

The authors are grateful to the editor and the two anonymous referees for their valuable comments which improved the quality of the paper. This work was supported by the Scientific Research Foundation of Chongqing University of Arts and Sciences (Grant No: R2013SC12), the National Natural Science Foundation of China (Grant Nos: 71271227, 11201505), and Program for Innovation Team Building at Institutions of Higher Education in Chongqing (Grant No: KJTD201321).

Received: 12 October 2013 Accepted: 25 February 2014 Published: 04 Mar 2014

\section{References}

1. Drury, SW, Liu, S, Lu, CY, Puntanen, S, Styan, GPH: Some comments on several matrix inequalities with applications to canonical correlations: historical background and recent developments. Sankhya, Ser. A 64, 453-507 (2002)

2. Gustafson, K: The geometrical meaning of the Kantorovich-Wielandt inequalities. Linear Algebra Appl. 296, 143-151 (1999)

3. Liu, SZ: Contributions to Matrix Calculus and Applications in Econometrics. Tinbergen Institute Research Series, vol. 106. Thesis Publishers, Amsterdam (1995)

4. Liu, SZ: Efficiency comparisons between the OLSE and the BLUE in a singular linear model. J. Stat. Plan. Inference 84, 191-200 (2000)

5. Rao, CR, Rao, MB: Matrix Algebra and Its Applications to Statistics and Econometrics. World Scientific, Singapore (1998)

6. Liu, SZ, Heyde, CC: Some efficiency comparisons for estimators from quasi-likelihood and generalized estimating equations. In: Moore, M, Froda, S, Léger, C (eds.) Mathematical Statistics and Applications: Festschrift for Constance van Eeden. Lecture Notes-Monograph Series, vol. 42, pp. 357-371. Inst. Math. Statist., Beachwood (2003)

7. Wang, SG, Ip, WC: A matrix version of the Wielandt inequality and its applications to statistics. Linear Algebra Appl. 296, 171-181 (1999)

8. Lu, CY: A generalized matrix version of the Wielandt inequality with some applications. Research Report, Department of Mathematics, North east Normal University, Changchun, China, 8 pp. (1999)

9. Liu, SZ, Lu, CY, Puntanen, S: Matrix trace Wielandt inequalities with statistical applications. J. Stat. Plan. Inference 139 2254-2260 (2009) 
10. Wang, LT, Yang, H: Matrix Euclidean norm Wielandt inequalities and their applications to statistics. Stat. Pap. 53, 521-530 (2012)

11. Marsaglia, G, Styan, GPH: Equalities and inequalities of ranks of matrices. Linear Multilinear Algebra 2, 269-292 (1974)

12. Groß, J: The general Gauss-Markov model with possibly singular dispersion matrix. Stat. Pap. 45, 311-336 (2004)

13. Anderson, TW: An Introduction to Multivariate Statistical Analysis, 3rd edn. Wiley, New York (2003)

14. Rao, CR: Least squares theory using an estimated dispersion matrix and its application to measurement of signals. In: Le Cam, LM, Neyman, J (eds.) Proceedings of the Fifth Berkeley Symposium on Mathematical Statistics and Probability (Berkeley, CA, 1965-66), vol. I: Statistics, pp. 355-372. University of California Press, Berkeley (1967)

15. Wang, SG, Yang, ZH: Pitman optimality of covariance-improved estimators. Chin. Sci. Bull. 40, 1150-1154 (1995)

10.1186/1029-242X-2014-110

Cite this article as: Wu and Yi: Matrix spectral norm Wielandt inequalities with statistical applications. Journal of Inequalities and Applications 2014, 2014:110

\section{Submit your manuscript to a SpringerOpen ${ }^{\circ}$ journal and benefit from:}

- Convenient online submission

- Rigorous peer review

- Immediate publication on acceptance

- Open access: articles freely available online

- High visibility within the field

- Retaining the copyright to your article 